\title{
Angioedema, An Unusual Reaction to Hair Dye
}

\section{Cristiana Ferreira*, Arminda Guilherme and Inês Lopes}

Department of Allergology, Centro Hospitalar Vila Nova de Gaia/Espinho, Portugal

*Corresponding author: Ferreira C, Department of Allergology, Centro Hospitalar Vila Nova de Gaia/Espinho, Portugal, Tel: +351 22786 5100; E-mail: cristianascferreira@gmail.com

Received: July 23, 2019; Accepted: August 04, 2019; Published: August 12, 2019

\section{Introduction}

Para-phenylendiamine (PPD) from hair dyes is known to be a potent skin sensitizer and it is responsible for most of the complications reported after the application of black henna dyes, which include contact dermatitis, scars, and temporary or permanent dyspigmentation. Less commonly, type 1 hypersensitivity reactions such as urticaria, angioedema, or anaphylaxis have been reported [1]. We present a case of allergic contact dermatitis with massive facial swelling owing to hair coloring with PPD.

\section{Case Description}

A 47-year-old woman, with a hypertension medicated with perindopril, presented to the emergency service with 2-day history of severe edema involving the upper and lower eyelids of both eyes and the scalp which developed overnight (FIG. 1). Patient was initially diagnosed as angioedema by Inhibitors of the Angiotensin Converting Enzyme and referred to our immunoallergology Department. She had used henna hair dye containing paraphenynlenediamine (PPD) the previous day. There was no history of previous sensitization with the use of hair dye or tattoos using black henna. The symptoms resolved completely with systemic corticosteroids and antihistamines in 2 weeks.

Patch testing was performed with the European/Portuguese baseline series and a hairdressing series (Vila Nova de Gaia, Roxall Aristegui, Portugal). Allergens were applied on the back with Finn Chambers (Chemotechnique Diagnostics, Vellinge, Sweden) on Scanpor tape (Bastos Viegas, Penafiel, Portugal).

Readings were performed on day (D) 2 and D3, according to ICDRG criteria, and showed strong positive reactions to pphenylenediamine (PPD) $1 \%$ pet., p-aminodiphenylamine $0.25 \%$ pet., n-isopropyl-N'-phenyl-p-phenylenediamine (IPPD) $0.1 \%$ pet., disperse orange nickel $1 \%$ pet, nickel $5 \%$ pet. (FIG. 2-3). Sensitization to PPD derivatives can cause crossreactions, our recommendations included the use of PPD-free hair dye or other para-dye ingredients. 


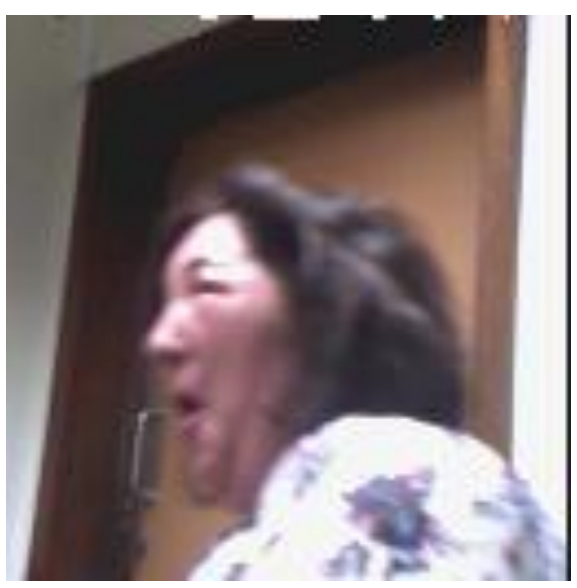

FIG. 1. Severe edema of eyelids and frontal area extending to the scalp.

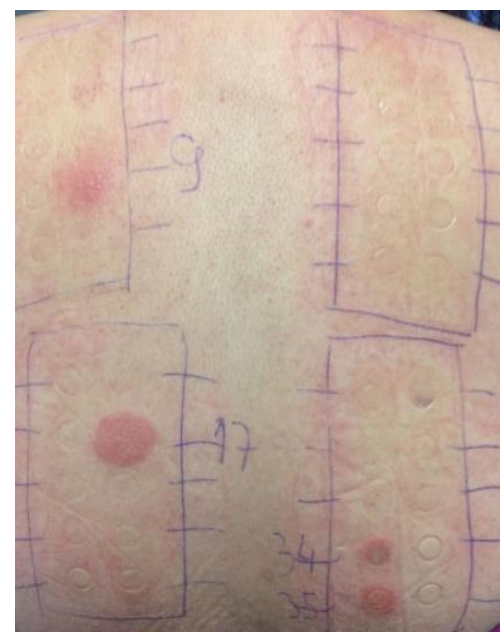

FIG. 2. The results of patch tests: A strong reaction (+++) to PPD, p-aminodiphenylamine, IPPD, disperse orange, nickel.

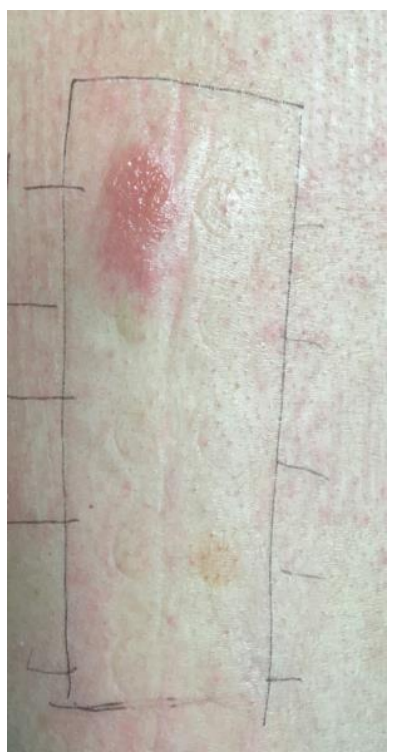

FIG. 3. Strong reaction (+++) p-aminodiphenylamine. 
www.yumedtext.com | August-2019

\section{Discussion}

This clinical case shows that although PPD is a common cause of allergic contact dermatitis, the presentation can be severe, including edema of the face and scalp which can be mistaken for angioedema.

In the present case, only a severe edema was prominent initially and misdiagnosis of angioedema was made in the emergency room, because no sign of dermatitis was present. Allergic contact dermatitis mimicking angioedema related to black henna has been reported before. This was a 23-year-old man who had a mild contact dermatitis to black henna tattoo with prior sensitization [2]. Contact-urticaria syndrome may be in the differential diagnosis for the hair dye allergic reactions which appears immediately upon contact with the causal agent [3]. We excluded this diagnosis because the reaction occurred one day after the hair dye exposure in our patient. Hence, patients who test positive to PPD should use semi-permanent hair dyes, which are PPD free [4-6].

\section{Conclusion}

It is important for the physicians to be aware of the differential diagnosis of a red swollen face and angioedema-like reactions should be included as these would require a different approach to the management. The learning points in this case highlight the fact that the differential diagnosis of facial swelling must also include contact dermatitis to hair products. Accurate diagnosis is needed for correct and prompt treatment and labelling of black henna should state the presence of PPD and the dangers linked with its use.

\section{REFERENCES}

1. Kluger N, Raison-Peyron N, Guillot B. Temporary henna tattoos: Sometimes serious side effects. Presse Med. 2008;37(7-8):1138-42.

2. Gokalp H, Kaya K. Angioedema-like allergic contact dermatitis related to black henna. Dermatol Online J. 2014;20(2).

3. Goossens A. Contact-allergic reactions to cosmetics. J Allergy (Cairo). 2011;2011:467071.

4. Robinson MK, Stotts J, Danneman PJ, et al. A risk assessment process for allergic contact sensitization. Food Chem Toxicol. 1989;27(7):479-9.

5. McFadden JP, Yeo L, White JL. Clinical and experimental aspects of allergic contact dermatitis to paraphenylenediamine. Clin Dermatol. 2011;29(3):316-24.

6. Evans C, Fleming JD. Allergic contact dermatitis from a henna tattoo. N Eng J Med. 2008;359:627. 